\title{
Erratum to: Hospital Readmission Among Medicaid Patients with an Index Hospitalization for Mental and/or Substance Use Disorder
}

\author{
Tami L. Mark, PhD, MBA \\ Karen Smoyer Tomic, PhD \\ Niranjana Kowlessar, PhD \\ Bong Chul Chu, PhD \\ Rita Vandivort-Warren, MSW \\ Shelagh Smith, MPH, CHES
}

An incomplete name was published for the first author. The complete name is Tami L. Mark.

Address correspondence to Tami L. Mark, PhD, MBA, Behavioral Health and Quality Research, Truven Health Analytics, Washington, DC 20008, USA. Phone: +1-301-2142211. Fax: +1-202-7197801. Email: tami.mark@truvenhealth.com.

Tami L. Mark, PhD, MBA, Truven Health Analytics, 7700 Old Georgetown Road, Ste GSO, Bethesda, MD 20814, USA. Phone: +1-301-2142211. Fax: +1-202-7197801. Email: tami.mark@truvenhealth.com

Niranjana Kowlessar, PhD, Truven Health Analytics, 7700 Old Georgetown Road, Ste GSO, Bethesda, MD 20814, USA.

Phone: 1-301-6283275. Email: nkowlessar@s-3.com

Bong Chul Chu, PhD, Truven Health Analytics, 7700 Old Georgetown Road, Ste GSO, Bethesda, MD 20814, USA. Phone: +1-805-6815877. Fax:+1-202-7197801. Email: bong-chul.chu@truvenhealth.com

Karen Smoyer Tomic, PhD, Oxford PharmaGenesis, Newtown, PA 18940, USA. Phone: +1-215-4979699. Email:

Karen.Tomic@pharmagenesis.com

Rita Vandivort-Warren, MSW, Health Resources and Services Administration, Rockville, MD, USA. Phone: +1-301-5944295.

Email: rvandivort-warren@hrsa.gov

Shelagh Smith, MPH, CHES, Substance Abuse and Mental Health Services Administration, Rockville, MD, USA. Phone: +1-301-2142211. Fax: +1-202-7197801. Email: tami.mark@truvenhealth.com

The online version of the original article can be found at http://dx.doi.org/10.1007/s11414-013-9323-5.

Journal of Behavioral Health Services \& Research, 2013. 273 (C) 2013 National Council for Behavioral Health. DOI 10.1007/s11414-013-9335-1 